\title{
A phase II study of irinotecan and pegylated liposomal doxorubicin in platinum-resistant recurrent ovarian cancer (Tohoku Gynecologic Cancer Unit 104 study)
}

\author{
Tadahiro Shoji ${ }^{1} \cdot$ Eriko Takatori $^{1} \cdot$ Hideo Omi $^{1} \cdot$ Masahiro Kagabu $^{1} \cdot$ Tatsuya Honda $^{2} \cdot$ Masayuki Futagami $^{3} \cdot$ \\ Yoshihito Yokoyama $^{3} \cdot$ Michiko Kaiho $^{4} \cdot$ Hideki Tokunaga $^{4} \cdot$ Takeo Otsuki $^{4} \cdot$ Tadao Takano $^{4} \cdot$ Nobuo Yaegashi ${ }^{4}$. \\ Takanobu Kojimahara ${ }^{5}$ - Tsuyoshi Ohta ${ }^{6} \cdot$ Satoru Nagase $^{6} \cdot$ Shu Soeda $^{7} \cdot$ Takafumi Watanebe $^{7}$. \\ Hiroshi Nishiyama ${ }^{8}$ Toru Sugiyama ${ }^{1}$
}

Received: 6 August 2016 / Accepted: 16 June 2017 / Published online: 27 June 2017

(C) The Author(s) 2017. This article is an open access publication

\begin{abstract}
Purpose We report a phase II clinical study of the combination of irinotecan (CPT-11) and pegylated liposomal doxorubicin (PLD) in platinum- and taxane-resistant recurrent ovarian cancer, based on the recommended doses determined in a phase I trial.

Methods PLD was administered intravenously at a dose of $30 \mathrm{mg} / \mathrm{m}^{2}$ on day 3 . CPT-11 was administered intravenously at a dose of $80 \mathrm{mg} / \mathrm{m}^{2}$ on days 1 and 15 , according to the recommendations of the phase I study. A single course of chemotherapy lasted 28 days, and patients underwent at least 2 courses until disease progression. The primary endpoint was antitumor efficacy, and the secondary endpoints were adverse events, progression-free survival (PFS), and overall survival (OS).

Results The response rate was $32.3 \%$ and the disease control rate was $64.5 \%$. Grade 3 and 4 neutropenia, anemia, and a decrease in platelet count were observed in $17(54.9 \%), 3(9.7 \%)$, and 1 patient $(3.2 \%)$, respectively. In terms of grade 3 or higher non-hematologic toxicities,
\end{abstract}

Tadahiro Shoji

tshoji@iwate-med.ac.jp

1 Department of Obstetrics and Gynecology, Iwate Medical University School of Medicine, 19-1 Uchimaru, Morioka 020-8505, Japan

2 Department of Obstetrics and Gynecology, Morioka Japanese Red Cross Hospital, 6-1-1 Sanbonyanagi, Morioka 020-8560, Japan

3 Department of Obstetrics and Gynecology, Hirosaki University School of Medicine, 53 Honcho, Hirosaki 036-8563, Japan

4 Department of Obstetrics and Gynecology, Tohoku University Graduate School of Medicine, 1-1 Seiryouchou, Sendai 980-8574, Japan grade 3 nausea occurred in 1 patient (3.2\%), vomiting in 3 patients (9.7\%), and grade 3 diarrhea and fatigue in 1 patient $(3.2 \%)$. The median PFS and OS rates were 2 months and not reached, respectively. Of the 11 patients with a treatment-free interval (TFI) of $\geq 3$ months, the response rate was $63.3 \%$, and the median PFS was 7 months.

Conclusions The treatment outcomes for the 31 patients enrolled in this study were unsatisfactory. However, subanalysis suggested that patients with a TFI of $\geq 3$ months had a good response rate and PFS. This suggests that CPT11/PLD combination therapy may be a chemotherapy option for platinum-resistant recurrent ovarian cancer.

Keywords Recurrent ovarian cancer · Chemotherapy · CPT-11 · PLD

Department of Obstetrics and Gynecology, Okitama Public General Hospital, 2000 Nishiotsuka, Kawanishimachi, Higashiokitamagun 992-0601, Japan

6 Department of Obstetrics and Gynecology, Faculty of Medicine, Yamagata University, 2-2-2 Iidanishi, Yamagata 990-9585, Japan

7 Department of Obstetrics and Gynecology, Fukushima Medical University School of Medicine, 1 Hikarigaoka, Fukushima 960-1295, Japan

8 Department of Obstetrics and Gynecology, Iwaki Kyouritsu Hospital, 16 Kusehara, Uchigomimayamachi, Iwaki 973-8555, Japan 


\section{Introduction}

In the treatment of patients with platinum-resistant recurrent ovarian cancer, it is essential to select drugs that do not demonstrate cross-resistance to the initial therapy. In Western countries, the type I DNA topoisomerase inhibitors such as topotecan [1], pegylated liposomal doxorubicin (PLD) [2], and gemcitabine [3] are used as monotherapies. In a Japanese phase II study of patients with ovarian cancer, previously treated with chemotherapy that included platinum-based agents, PLD was reported to achieve an overall response rate of $21.9 \%(27.3 \%$ [3/11] in the platinum-sensitive group and $21.0 \%$ [13/62] in the platinum-resistant group) [2]. In a phase III noninferiority study comparing PLD with topotecan, PLD achieved an overall response rate of $19.7 \%$, a median progression-free survival (PFS) of 16.1 weeks, and a mean survival time (MST) of 60.0 weeks; in particular, in patients with platinum-resistant tumors, the response rate was $12.3 \%$, the median PFS was 9.1 weeks, and the MST was 35.6 weeks [4]. These results suggested that PLD could be a promising therapeutic agent for recurrent ovarian cancer. On the other hand, irinotecan hydrochloride (CPT-11), an anticancer agent developed in Japan, acts by inhibiting topoisomerase I. In a study of platinum-resistant recurrent ovarian cancer treated with CPT-11 monotherapy $\left(100 \mathrm{mg} / \mathrm{m}^{2}\right)$, the response rate was $29 \%$; the tumor growth inhibition rate (complete response $[\mathrm{CR}]+$ partial response $[\mathrm{PR}]+$ no change) was $61 \%$; the median time to progression was 17 weeks; and the MST was 8 months, exhibiting favorable results [5]. Sugiyama et al. reported that CPT-11/cisplatin combination therapy was effective as a second-line chemotherapy for recurrent ovarian cancer after treatment with a platinum agent [5], which means that CPT-11 may be effective for platinumand taxane-resistant tumors.

We previously conducted a phase I study of the combination of CPT-11 and PLD in patients with platinum- and taxane-resistant recurrent ovarian cancer and reported that the recommended doses of CPT- 11 and PLD were 80 and $30 \mathrm{mg} / \mathrm{m}^{2}$, respectively [6]. Here, we report a phase II study based on the recommended doses determined in the phase I study.

\section{Subjects and methods}

\section{Study population}

Approval was obtained from the intramural ethics committee of each study center. A multi-center clinical study was conducted in patients with recurrent ovarian cancer who were enrolled in the study between April 2013 and March 2015 and who met the following criteria: (1) ovarian cancer confirmed by histological or cytological diagnosis, (2) recurrence $<6$ months after previous chemotherapy, (3) a measurable or evaluable lesion (including CA 125 level), (4) European Oncology Cooperative Group performance status 0-2, (5) age between 20 and 75 years, (6) expected survival time of $>2$ months, (7) no effect on major organ functions (white blood cell count $\geq 3000 / \mathrm{mm}^{3}$, neutrophil count $\geq 1500 / \mathrm{mm}^{3}$, platelet count $\geq 10,0000 / \mathrm{mm}^{3}$, total bilirubin $\leq 1.5 \mathrm{mg} / \mathrm{dL}$ ), and (8) informed consent provided. Exclusion criteria were: (1) serious complication(s), (2) evident pulmonary fibrosis or interstitial pneumonitis, (3) pleural or cardiac effusion necessitating prompt local treatment, (4) brain metastasis requiring prompt treatment, (5) diarrhea (watery stool), (6) intestinal paralysis or intestinal obstruction, (7) active infection requiring treatment with antimicrobial agents, and (8) patients considered inappropriate as subjects by the physician in charge for any other reason.

\section{Protocol}

PLD was administered intravenously at a dose of $30 \mathrm{mg} / \mathrm{m}^{2}$ on day 3. CPT-11 was administered intravenously at a dose of $80 \mathrm{mg} / \mathrm{m}^{2}$ on days 1 and 15 , according to the recommendation of the phase I study. A single course of chemotherapy lasted 28 days and, as a general rule, patients underwent at least two courses, until progressive disease (PD).

\section{Sample size}

In previous studies of patients with platinum-resistant recurrent ovarian cancer, the response rates associated with multiple-agent chemotherapy with CPT-11 ranged from 29 to $50 \%[7,8,9]$. On the other hand, PLD monotherapy was reported to have response rates between 8.3 and $21.9 \%$ [2, 10, 11]. If the CPT-11/PLD combination therapy resulted in a response rate well below that of the above therapies, it would be assumed that the combination therapy had no clinical significance. Therefore, we set the expected response rate and the threshold response rate of the CPT$11 /$ PLD combination therapy in this study at 40 and $20 \%$, respectively. The required sample size was calculated as 36 using a double-sided $\alpha$ error of 0.05 and $\beta$ error of 0.2 . The planned sample size was estimated at 40 , including patients who might become ineligible.

\section{Criteria for changing the dosing schedule}

If any of the following conditions applied, CPT-11 administration on day 15 was postponed: (1) white blood cell count $\leq 2000 / \mathrm{mm}^{3}$, (2) neutrophil count $\leq 1000 / \mathrm{mm}^{3}$, (3) 
platelet count $\leq 75,000 / \mathrm{mm}^{3}$, or (4) grade 1 or higher diarrhea. Once recovery was confirmed, the drug was administered on day 22. If recovery from the condition was not seen on day 22, the second CPT-11 administration was skipped (not to be administered on day 29). The criteria for proceeding to the second and subsequent courses were: (1) white blood cell count $\geq 3000 / \mathrm{mm}^{3}$, (2) neutrophil count $\geq 1500 / \mathrm{mm}^{3}$, (3) platelet count $\geq 100,000 / \mathrm{mm}^{3}$, (4) total bilirubin $\leq 1.5 \mathrm{mg} / \mathrm{dL}$, (5) diarrhea grade 0 , and (6) grade 1 or lower hand-foot syndrome and mucositis. If the patient met any of the above criteria, treatment was postponed for up to 14 days for recovery. If, after 14 days, recovery from the above conditions was not observed (4 weeks for hand-and-foot syndrome or stomatitis), the treatment was discontinued. If the severity of hand-foot syndrome or mucositis remained at grade 2 or higher after a 14-day postponement, PLD on day 3 in the next treatment course was skipped.

\section{Criteria for dose reduction}

The doses of CPT-11 and PLD in the next course were reduced according to the severity of any adverse reactions that occurred in the previous course. If grade 4 leukopenia, grade 4 neutropenia, or grade 3 thrombocytopenia was observed in the preceding course, the CPT-11 dosage was reduced by $10 \mathrm{mg} / \mathrm{m}^{2}$ and PLD was reduced by $7.5 \mathrm{mg} / \mathrm{m}^{2}$. If grade 2 or higher diarrhea, spasmodic abdominal pain, or watery stool was observed, the CPT-11 dose was reduced by $10 \mathrm{mg} / \mathrm{m}^{2}$. If grade 3 hand-foot syndrome or mucositis was observed, the PLD dose was reduced by $7.5 \mathrm{mg} /$ $\mathrm{m}^{2}$, regardless of whether or not these conditions improved before the start of the next course.

\section{Endpoints/variables}

The primary endpoint was antitumor efficacy, and the secondary endpoints were adverse events, PFS, and overall survival (OS). The antitumor effect was evaluated via imaging after every two courses. For evaluation of the antitumor effect, the best response rate was calculated according to the RECIST (Response Evaluation Criteria in Solid Tumors) version 1.0 guidelines. Adverse events were evaluated according to the National Cancer Institute Common Toxicity Criteria version 4.0.

\section{Statistical analysis}

PFS and OS were calculated from the chemotherapy start date to the documented date of progression, death, or last follow-up, whichever occurred first. Impact on survival was assessed by constructing Kaplan-Meier curves with a log-rank test. The Chi-square test and the log-rank test were used to compare and analyze patient characteristics between the phase I and II groups, and between patients with a $<3$ - and $\geq 3$-month treatment-free interval (TFI). All reported significance values were two tailed at a level of 0.05 .

\section{Results}

\section{Patient background characteristics}

Table 1 shows the background characteristics of the 31 patients enrolled in this study from April 2013 through March 2015. All patients had been treated with taxane- or platinum-based agents as part of their previous therapy. Twenty patients $(64.5 \%)$ had a TFI $<3$ months.

Table 1 Patient characteristics $(N=31)$

\begin{tabular}{|c|c|}
\hline \multicolumn{2}{|l|}{ Age (year) } \\
\hline Median & 56 \\
\hline Range & $38-74$ \\
\hline \multicolumn{2}{|l|}{ PS } \\
\hline 0 & 25 \\
\hline 1 & 6 \\
\hline \multicolumn{2}{|l|}{ FIGO stage } \\
\hline I & 3 \\
\hline II & 0 \\
\hline III & 20 \\
\hline IV & 8 \\
\hline \multicolumn{2}{|l|}{ Histological type } \\
\hline Serous & 20 \\
\hline Mucinous & 1 \\
\hline Clear cell & 8 \\
\hline Unknown & 2 \\
\hline \multicolumn{2}{|l|}{ Previous regimens } \\
\hline 1 & 14 \\
\hline 2 & 10 \\
\hline $3 \leq$ & 7 \\
\hline \multicolumn{2}{|l|}{ Last regimen } \\
\hline Paclitaxel/carboplatin & 23 \\
\hline Docetaxel/carboplatin & 3 \\
\hline Docetaxel/gemcitabine & 2 \\
\hline Gemcitabine & 2 \\
\hline Paclitaxel & 1 \\
\hline \multicolumn{2}{|l|}{ TFI (months) } \\
\hline$<3$ & 20 \\
\hline $3 \leq, 6<$ & 11 \\
\hline
\end{tabular}

$T F I$ treatment-free interval 


\section{Antitumor effect}

Table 2 shows the antitumor effects of the treatment. Partial response (PR), stable disease (SD), and progressive disease (PD) were observed in 10 (32.3\%), $10(32.3 \%)$, and $11(35.5 \%)$ patients, respectively. The response rate was $32.3 \%$ and the disease control rate

Table 2 Response

\begin{tabular}{lcc}
\hline & $N$ & $\%$ \\
\hline CR & 0 & 0 \\
PR & 10 & 32.3 \\
SD & 10 & 32.3 \\
PD & 11 & 35.5 \\
Overall response & 10 & 32.3 \\
Disease control & 20 & 64.5 \\
\hline
\end{tabular}

$C R$ complete response, $P R$ partial response, $S D$ stable disease, $P D$ progressive disease

Table 3 Toxicity

Grade 1 Grade 2 Grade 3 Grade 4 Grade $3 \leq(\%)$

\begin{tabular}{lrrrrl}
\hline Hematologic toxicity & & & & \\
Leucopenia & 2 & 14 & 8 & 2 & $10(32.3)$ \\
Neutropenia & 0 & 7 & 11 & 6 & $17(54.8)$ \\
Anemia & 13 & 11 & 3 & 0 & $3(9.7)$ \\
$\begin{array}{l}\text { Thrombocy- } \\
\text { topenia }\end{array}$ & 6 & 1 & 1 & 0 & $1(3.2)$ \\
$\begin{array}{l}\text { Non-hematologic toxicity } \\
\text { Nausea }\end{array}$ & 16 & 3 & 1 & 0 & $1(3.2)$ \\
Vomiting & 6 & 1 & 3 & 0 & $3(9.7)$ \\
Diarrhea & 1 & 2 & 1 & 0 & $1(3.2)$ \\
Hand-foot & 3 & 3 & 0 & 0 & $0(0)$ \\
Mucositis & 12 & 1 & 0 & 0 & $0(0)$ \\
Appetite & 10 & 7 & 0 & 0 & $0(0)$ \\
$\quad$ loss & & & & & \\
Fatigue & 8 & 3 & 1 & 0 & $1(3.2)$ \\
\hline
\end{tabular}

was $64.5 \%$. However, no patient achieved complete response (CR).

\section{Adverse events}

Grades 3 and 4 neutropenia were observed in $11(35.5 \%)$ and $6(19.4 \%)$ patients, respectively. Grade 3 anemia was observed in 3 patients $(9.7 \%)$. A grade 3 decrease in platelet count was observed in 1 patient $(3.2 \%)$. In terms of grade 3 or higher non-hematologic toxicities, grade 3 nausea occurred in 1 patient (3.2\%), vomiting in 3 patients $(9.7 \%)$, and grade 3 diarrhea and fatigue in $1(3.2 \%)$ patient (Table 3).

\section{Administration status}

In total, 119 treatment courses were administered to the 31 patients. Over the course of the study, 19 treatment cycles were postponed $(16.0 \%)$. The reasons for postponement were as follows: neutrophil count $<1500 / \mathrm{mm}^{3}$ ( 15 cycles); grade 2 hand-foot syndrome ( 1 cycle); and the discretion of the attending physician ( 3 cycles).

CPT-11 on day 15 was postponed in 3 cycles $(2.5 \%)$. The reasons for postponement were neutrophil count $<1000 / \mathrm{mm}^{3}$ ( 2 cycles) and the discretion of the attending physician (1 cycle). CPT-11 on day 15 was skipped in 5 cycles $(4.2 \%)$. The reasons included lack of neutrophil count recovery ( 1 cycle), lack of platelet count recovery (1 cycle), and the discretion of the attending physician (3 cycles). CPT-11 and PLD doses were reduced in 1 patient owing to grade 4 neutropenia in the previous course. Grade 4 neutropenia was also observed in 5 other patients; all 5 patients experienced PD and their regimens were changed.

\section{PFS and OS}

Median PFS was 2 months (95\% confidence interval [CI] 2-6) and PFS at 1 year was $12.9 \%$ (95\% CI 4.9-29.7) (Fig. 1a). The median OS was not reached, and the survival rate at 1 year was $63.6 \%$ (95\% CI 45.4-78.6) (Fig. 1b).
Fig. 1 Survival. a Progressionfree survival. b Overall survival
A

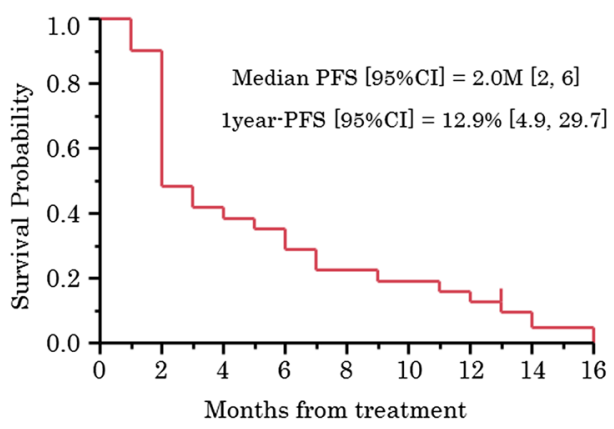

B

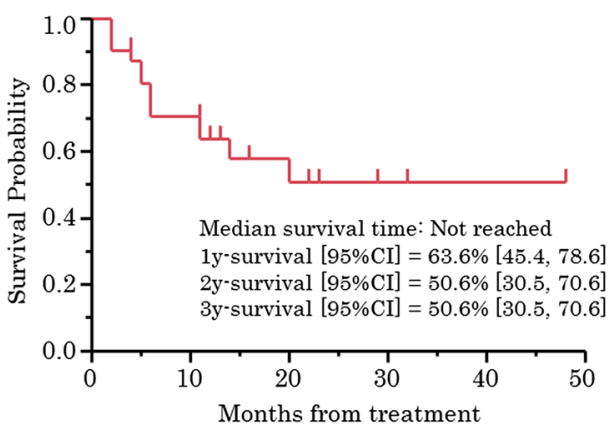




\section{Discussion}

We previously reported that the response rate and disease control rate of platinum-resistant recurrent ovarian cancer to CPT-11/VP16 combination therapy were 50.0 and $83.3 \%$, respectively [9]. In a phase I study of 12 patients, the response rate and disease control rate were $58.3 \%$ (including 1 patient who achieved CR) and $75.0 \%$, respectively [6]. Of the 31 patients in the present phase II study, the response rate and disease control rate were 32.2 and $64.5 \%$, respectively, which did not meet our expectations. For further analysis, we compared the backgrounds between the 12 patients enrolled in the phase I study and the 31 patients enrolled in the present phase II study. Between the two patient groups, there were no differences in the proportion of PS 1, incidence of clear cell carcinoma, or the proportion of patients treated with $\geq 3$ regimens. A significant difference was observed between the two groups regarding the proportion of patients with a TFI of $<3$ months (Table 4). We, therefore, compared the results between patients with a TFI $<3$ months and those with a TFI of 3-6 months in the present study. The comparisons between the 20 patients with a TFI of $<3$ months and the 11 patients with a TFI of 3-6 months showed that the response rates were 15.0 and $63.3 \%$, respectively; the disease control rates were 55.0 and $81.8 \%$, respectively; the median PFS rates were 2 months $(95 \%$ CI 2-3) and 7 months (95\% CI $2-13)$, respectively $(P=0.039$, hazard ratio [HR] 0.48); and the 2-year survival rates were $36.4 \%$ (95\% CI 16.1-63.0) and 80.8\% (95\% CI 47.2-95.2), respectively $(P=0.092$, HR 0.29$)$ (Table 5$)$. We hypothesized that the large differences in the response, PFS, and OS rates between the phase I and phase II studies may be related to the differences in TFI between the two groups: in the phase I study, 4 patients $(25.0 \%)$ had a TFI $<3$ months, while 20 patients $(64.5 \%)$ in the phase II study had a TFI $<3$ months. Therefore, the proportion of patients with a TFI of $<3$ months was higher in the phase II study. Based on these results, we consider that CTP-11/PLD combination therapy can suppress tumor progression in patients

Table 4 Difference of the background in phase I and II study

\begin{tabular}{llcc}
\hline & Phase I $(N=12)$ & Phase II $(N=31)$ & $P$ value \\
\hline $\begin{array}{l}\text { ECOG performance } \\
\text { status } 1^{*}\end{array}$ & 1 & 6 & 0.37 \\
Clear cell carcinoma* & 3 & 8 & 0.92 \\
TFI <3 month* & 4 & 20 & 0.04 \\
$\begin{array}{l}\text { Previous regimens } \\
3 \leq *\end{array}$ & 5 & 7 & 0.21 \\
\hline
\end{tabular}

TFI treatment-free interval

* Chi-square test
Table 5 Comparison of treatment results due to the difference in the TFI

\begin{tabular}{llll}
\hline TFK (months) & $<3(N=20)$ & $3<,<6(N=11)$ & $P$ value \\
\hline Response rate $(\%)^{*}$ & 15.0 & 63.3 & 0.0007 \\
$\begin{array}{c}\text { Disease control rate } \\
(\%)^{*}\end{array}$ & 55.0 & 81.8 & 0.016 \\
$\begin{array}{c}\text { Median PFS }(95 \% \mathrm{CI}) \\
\quad 2[2,3]\end{array}$ & $7[2,11]$ & 0.039 \\
$\begin{array}{c}\text { 2-yonths)** } \\
(95 \% \mathrm{CI})(\%)^{* *}\end{array}$ & $36.4(16.1,63.0)$ & $80.8(47.2,95.2)$ & 0.092 \\
\hline
\end{tabular}

TFI treatment-free interval, $P F S$ progression-free survival

* Chi-square test, ** log-rank test

with a TFI of 3-6 months, and may represent a secondline chemotherapy treatment option for platinum-resistant recurrent ovarian cancer, because it is associated with only mild adverse drug reactions. To confirm the treatment efficacy, further studies of its efficacy in platinum-resistant recurrent ovarian cancer with a TFI of 3-6 months, after excluding refractory cases, are necessary. The results of a questionnaire survey administered to member institutions of the Japanese Gynecologic Oncology Group (JGOG) in 2014 showed that PLD monotherapy was used as secondline therapy for platinum-resistant recurrent ovarian cancer in $47 \%$ of these institutions. Therefore, we advocate conducting a future phase III study, based on the results of this phase II study, on a nationwide basis in cooperation with societies such as the JGOG. It is also our view that the usefulness of CPT-11/PLD combination therapy should be verified by a superiority trial comparing PLD monotherapy as a control arm and CPT-11/PLD combination therapy as an experimental arm.

This 2-year study included only 31 patients, although the target sample size was 40 . The small sample size was at least partly attributable to the difficulty in registering patients in some institutions. We presumed that approximately 5 patients with platinum-resistant recurrent ovarian cancer would attend each institution annually; however, no patients enrolled in 3 of the 7 institutions participating in the TGCU (Tohoku Gynecologic Cancer Unit) study group. This might have been due to the shortage of obstetricians and gynecologists in Japan, where many of them have to work very hard and they are very busy. We need to be more aware of the importance of clinical studies and greater efforts to establish scientific evidence are needed. Another limitation of this study was the cost of the clinical data management center. We realized that we needed to extend the registration period to reach the target sample size of 40 patients, but we decided to terminate the study owing to the cost of the extension. This is not a particular problem for our study group (TGCU). In Japan, clinical trials are being conducted in collaboration with clinical data management 
centers to ensure the quality of the studies. Since the cost of clinical data management centers is a huge expense, securing finance for projects is an important issue that needs to be resolved.

Recent studies have reported the use of molecular targeted drugs for treatment of platinum-resistant recurrent ovarian cancer. In the AURELIA (Avastin Use in Platinum-Resistant Epithelial Ovarian Cancer) trial, the median PFS of single-agent chemotherapy with bevacizumab was 6.7 months, and the HR was 0.48 , compared with singleagent chemotherapy alone [12]. However, the molecular targeted drugs such as bevacizumab are expensive and pose a significant financial burden on patients. In Japan, conventional anticancer drugs have an advantage over molecular targeted drugs from a health economics perspective. For example, if a regimen used in the AURELIA trial, paclitaxel plus bevacizumab, PLD plus bevacizumab, or topotecan plus bevacizumab, was administered to a patient with a height of $160 \mathrm{~cm}$, weight of $60 \mathrm{~kg}$ and body surface area of $1.6 \mathrm{~m}^{2}$, for 6 cycles, it would cost approximately 34,000 dollars $(2,800,000$ Japanese yen), 60,000 dollars $(4,900,000$ Japanese yen), or 43,000 dollars $(3,600,000$ Japanese yen), respectively. In contrast, CPT-11/PLD combination therapy (for 6 cycles) costs approximately 24,000 dollars (2,000,000 Japanese yen). We consider that CPT-11/ PLD combination therapy can contribute to the reduction of health costs. Given that platinum-resistant recurrent ovarian cancer cannot be completely cured, chemotherapy has an important role in maintaining the quality of life and reducing the financial burden of patients. More recently, antiPD-1 antibodies have become a treatment option in ovarian cancer, in addition to molecular targeted drugs such as olaparib [13], trebananib [14], and pazopanib [15]. Hamanishi et al. reported that the response rate and disease control rate of nivolumab in platinum-resistant recurrent ovarian cancer were 15 and $45 \%$, respectively [16]. Following this pioneer study, a clinical trial of an anti-PD-1 antibody is currently being conducted in Japan. However, like molecular targeted drugs, anti-PD-1 antibodies are very expensive. CPT-11/PLD combination therapy has both cost and safety (less severe adverse drug reactions) advantages. We recommend that the usefulness of anticancer drugs should be reevaluated from economical and safety perspectives.

Acknowledgements This clinical trial was performed as TGCU study. We thank all TGCU investigators.

\section{Compliance with ethical standards}

Funding This study was funded by Tohoku Gynecologic Cancer Unit (TGCU), Morioka, Japan.

Conflict of interest The authors have no conflicts of interest to declare.
Human and animal rights This article does not contain any studies with animals performed by any of the authors.

Ethical approval All procedures performed in studies involving human participants were in accordance with the ethical standards of the institutional research committee and with the 1964 Declaration of Helsinki and its later amendments or comparable ethical standards.

Informed consent Written informed consent was obtained from all study participants.

Open Access This article is distributed under the terms of the Creative Commons Attribution 4.0 International License (http://creativecommons.org/licenses/by/4.0/), which permits unrestricted use, distribution, and reproduction in any medium, provided you give appropriate credit to the original author(s) and the source, provide a link to the Creative Commons license, and indicate if changes were made.

\section{References}

1. Aoki D, Katsumata N, Nakanishi T, Kigawa J, Fujiwara K, Takehara K, Kamiura S, Hiura M, Hatae M, Sugiyama T, Ochiai K, Noda K (2011) A phase II clinical trial of topotecan in Japanese patients with relapsed ovarian carcinoma. Jpn J Clin Oncol 41:320-327

2. Katsumata N, Fujiwara Y, Kamura T, Nakanishi T, Hatae M, Aoki D, Tanaka K, Tsuda H, Kamiura S, Takehara K, Sugiyama T, Kigawa J, Fujiwara K, Ochiai K, Ishida R, Inagaki M, Noda K (2008) Phase II clinical trial of pegylated liposomal doxorubicin (JNS002) in Japanese patients with mullerian carcinoma (epithelial ovarian carcinoma, primary carcinoma of fallopian tube, peritoneal carcinoma) having a therapeutic history of platinumbased chemotherapy: a phase II study of the Japanese Gynecologic Oncology Group. Jpn J Clin Oncol 38:777-785

3. Yoshino K, Hiramatsu K, Enomoto T, Fujita M, Ueda Y, Kimura T, Kobayashi E, Kiyohara Y, Tsutsui T, Kimura T (2012) Salvage chemotherapy using gemcitabine for taxane/platinum-resistant recurrent ovarian cancer: a single institutional experience. Anticancer Res 32:4029-4033

4. Gordon AN, Fleagle JT, Guthrie D, Parkin DE, Gore ME, Lacave AJ (2001) Recurrent epithelial ovarian carcinoma: a randomized phase III study of pegylated liposomal doxorubicin versus topotecan. J Clin Oncol 19:3312-3322

5. Matsumoto K, Katsumata N, Yamanaka Y, Yonemori K, Kohno T, Shimizu C, Andoh M, Fujiwara Y (2006) The safety and efficacy of the weekly dosing of irinotecan for platinum- and taxanesresistant epithelial ovarian cancer. Gynecol Oncol 100:412-416

6. Sugiyama T, Yakushiji M, Nishida T, Ushijima K, Okura N, Kigawa J, Terakawa N (1998) Irinotecan (CPT-11) combined with cisplatin in patients with refractory or recurrent ovarian cancer. Cancer Lett 128:211-218

7. Shoji T, Takatori E, Kaido Y, Omi H, Yokoyama Y, Mizunuma H, Kaiho M, Otsuki T, Takano T, Yaegashi N, Nishiyama H, Fujimori K, Sugiyama T (2014) A phase I study of irinotecan and pegylated liposomal doxorubicin in recurrent ovarian cancer (Tohoku Gynecologic Cancer Unit 104 study). Cancer Chemother Pharmacol 73:895-901

8. Nishio S, Sugiyama T, Shouji T, Yoshizaki A, Kitagawa R, Ushijima K, Kamura T (2007) Pilot study evaluating the efficacy and toxicity of irinotecan plus oral etoposide for platinum- and 
taxane-resistant epithelial ovarian cancer. Gynecol Oncol 106:342-347

9. Shoji T, Takatori E, Omi H, Kumagai S, Yoshizaki A, Yokoyama Y, Mizunuma H, Fujimoto T, Takano T, Yaegashi N, Tase T, Nakahara K, Kurachi H, Nishiyama H, Sugiyama T (2011) Phase II clinical study of the combination chemotherapy regimen of irinotecan plus oral etoposide for the treatment of recurrent ovarian cancer (Tohoku Gynecologic Cancer Unit 101 Group Study). Int J Gynecol Cancer 21:44-50

10. Mutch DG, Orlando M, Goss T, Teneriello MG, Gordon AN, McMeekin SD, Wang Y, Scribner DR Jr, Marciniack M, Naumann RW, Secord AA (2007) Randomized phase III trial of gemcitabine compared with pegylated liposomal doxorubicin in patients with platinum-resistant ovarian cancer. J Clin Oncol 25:2811-2818

11. Gordon AN, Granai CO, Rose PG, Hainsworth J, Lopez A, Weissman C, Rosales R, Sharpington T (2000) Phase II study of liposomal doxorubicin in platinum- and paclitaxel-refractory epithelial ovarian cancer. J Clin Oncol 18:3093-3100

12. Pujade-Lauraine E, Hilpert F, Weber B, Reuss A, Poveda A, Kristensen G, Sorio R, Vergote I, Witteveen P, Bamias A, Pereira D, Wimberger P, Oaknin A, Mirza MR, Follana P, Bollag D, Ray-Coquard I (2014) Bevacizumab combined with chemotherapy for platinum-resistant recurrent ovarian cancer: the AURELIA open-label randomized phase III trial. J Clin Oncol 32:1302-1308

13. Ledermann J, Harter P, Gourley C, Friedlander M, Vergote I, Rustin G, Scott CL, Meier W, Shapira-Frommer R, Safra T,
Matei D, Fielding A, Spencer S, Dougherty B, Orr M, Hodgson D, Barrett JC, Matulonis U (2014) Olaparib maintenance therapy in patients with platinum-sensitive relapsed serous ovarian cancer: a preplanned retrospective analysis of outcomes by BRCA status in a randomised phase 2 trial. Lancet Oncol 15:852-861

14. Monk BJ, Poveda A, Vergote I, Raspagliesi F, Fujiwara K, Bae DS, Oaknin A, Ray-Coquard I, Provencher DM, Karlan BY, Lhommé C, Richardson G, Rincón DG, Coleman RL, Herzog TJ, Marth C, Brize A, Fabbro M, Redondo A, Bamias A, Tassoudji M, Navale L, Warner DJ, Oza AM (2014) Anti-angiopoietin therapy with trebananib for recurrent ovarian cancer (TRINOVA-1): a randomised, multicentre, double-blind, placebo-controlled phase 3 trial. Lancet Oncol 15:799-808

15. Kim JW, Mahner S, Wu LY, Shoji T, Kim BG, Zhu JQ, Takano T, Park SY, Kong BH, Wu Q, Wang KL, Ngan HY, Liu JH, Wei LH, Mitrica I, Zhang P, Crescenzo R, Wang Q, Cox CJ, Harter P, du Bois A (2015) Pazopanib maintenance therapy in East Asian Women with advanced epithelial ovarian cancer: results from AGO-OVAR16 and an East Asian Study. Int J Gynecol Cancer (epub ahead of print)

16. Hamanishi J, Mandai M, Ikeda T, Minami M, Kawaguchi A, Murayama T, Kanai M, Mori Y, Matsumoto S, Chikuma S, Matsumura N, Abiko K, Baba T, Yamaguchi K, Ueda A, Hosoe Y, Morita S, Yokode M, Shimizu A, Honjo T, Konishi I (2015) Safety and antitumor activity of anti-PD-1 antibody, nivolumab, in patients with platinum-resistant ovarian cancer. J Clin Oncol 33:4015-4022 